\title{
Nanolipid carrier-based thermoreversible gel for localized delivery of docetaxel to breast cancer
}

\author{
Tanya Vohra • Inderpreet Kaur • Hemraj Heer • \\ Rayasa Ramachandra Murthy
}

Received: 30 July 2012 /Revised: 19 November 2012 / Accepted: 28 January 2013 /Published online: 16 March 2013

(C) Springer-Verlag Wien 2013

\begin{abstract}
Intratumoral and intralesional administration of anticancer drugs in gels and implantable formulations is gaining much importance on account of its advantage of site-specific delivery with highly dependable freedom from unwanted side effects. Nanolipid carriers (NLC) are the preferred vehicle due to their improved properties particularly drug loading. In the present investigation, glyceryl monostearate-oleic acid NLCs loaded with docetaxel were prepared by emulsification and ultrasonication technique and were incorporated in thermoreversible pluronic F127 gel (TRPgel) for intralesion injection to breast tissue. The NLCs were spherical particles of $113 \mathrm{~nm}$ size with a negative zeta potential of -32.8 and $85 \%$ drug entrapment. In vitro drug release profile of the NLC showed $96 \%$ drug release in $48 \mathrm{~h}$ following Higuchi release kinetics. NLC incorporated TRPgel showed mucoadhesive force of 3.07 dynes $/ \mathrm{cm}^{2}$ and gelling temperature in the range of 32 to $37{ }^{\circ} \mathrm{C}$. The drug entrapped gel was also subjected to in vitro cytotoxicity study in human B-16 and HeLa cell lines by 3-(4,5-dimethylthiazol-2-yl)-2,5-diphenyltetrazolium bromide (MTT) assay and in vivo drug distribution study in breast tissue in healthy Wistar rats. The MTT assay revealed that docetaxel-loaded NLC incorporated into gel showed lower cytotoxicity than docetaxel. However, in vivo breast tissue distribution studies showed high tissue drug concentration, sustained over a period of $60 \mathrm{~h}$ in comparison to docetaxel and docetaxel-loaded NLCs. These results suggest that nanolipid carrier of docetaxel in TRPgel could be a promising carrier system to deliver drug to tumor by intralesional administration for improving therapeutic benefits of docetaxel.
\end{abstract}

T. Vohra $(\bowtie) \cdot$ I. Kaur $\cdot$ H. Heer $\cdot$ R. R. Murthy $(\bowtie)$

Nano Medicine Research Center, ISF College of Pharmacy,

Moga 142001 Punjab, India

e-mail: vohratanya4@gmail.com

e-mail: m_rsr@rediffmail.com
Keywords Docetaxel · Thermoreversible gels $\cdot$ Nanolipid carriers · Breast cancer · Pluronic F127

\section{Introduction}

Breast cancer accounts for $33 \%$ of all incident cancers in women, with an increasing mortality rate in North America (Jemal et al. 2005). Current clinical strategies dealing with these solid tumors primarily consist of surgical excision, irradiation, and chemotherapy. But the severity of increase and occurrence of tumors leads to adjuvant therapy of chemotherapeutic drugs (Rouzier et al. 2001). Most cytotoxic agents when administered systemically into cancer patients provide various limitations and challenges. These limitations include large volume of distribution leading to systemic toxicity of vital organs, low blood flow into interior of a tumor site resulting in inability to provide optimal dose, and frequent dose reduction due to various toxicities like hematologic, neurologic, and physiological. For these reasons, drug delivery technology research has focused on targeting anticancer drugs to a specific site or to develop intratumoral or intralesional injections to provide timed release profile for better management and cure of cancer (Rob et al. 2006).

Nanolipid carriers (NLCs) are considered a smarter generation of nanoparticles which possesses improved properties for drug loading, modulation of the delivery profile, excellent biocompatibility, and easy modulation of biodegradation time in vivo (Muller et al. 2007). NLC are promising carrier to increase the prolonged drug residence in the target organ which can extend exposure of tumor cells to antitumor drug. Mendes et al. (2009), based on his experiments, reported that intralesional injection of anti cancer agents is a promising approach for drug targeting in neoadjuvant chemotherapy in breast cancer treatment. The importance of intramammary injection in the regression of 
human breast cancer xenografts growing bilaterally in nude mice and potentiation of local antitumor actions of interferons (IFNs) by recombinant human tumor necrosis factor $\alpha$ was reported by Luciano et al. (1995). The structure of NLC is irregular and has defects in the packing of molecules which offers maximum space to accommodate drug molecules resulting in high drug loading capability (Li et al. 2010).

Injectable in situ gels have caused much attention and have been studied quite a lot in the fields of drug delivery, possibly due to their ease of preparation, ease of control in quality, nonuse of organic solvents, site-specific delivery, prolonged action periods, and improved patient compliance (Matsumura and Maeda 1986). The in situ gel system may be $\mathrm{pH}$-sensitive, ion-sensitive, or thermosensitive, according to the different materials used. Thermosensitive gel approach can be advantageous for particular application as it is in sol form when administered through various routes like subcutaneous, topical, intralesional, etc. and gets transformed to gel state at body temperature. Thermosensitive gel made of pluronics 127 known as OncoGel is example of drug delivery technology that uses both physical targeting to the target body site and controlled release of drug. Pluronic F127 (PF127) can form a gel with good thermosensitivity. It is usually regarded as nontoxic and has been applied in localized drug delivery such as intramuscular, intraperitoneal, and subcutaneous injections (Wang and Johnston 1995; Liu et al. 2007).

The overall aim of the current study was to develop a simple and generally applicable intratumoral injection strategy for developing an effective way to treat breast cancers through intralesional administration into these solid tumors. NLCs loaded with docetaxel were prepared and were incorporated in thermoreversible pluronic F127 gel (TRPgel). The NLCs were characterized for morphology, particle size, surface charge, entrapment efficiency, and for in vitro drug release profile. NLC-incorporated TRPgel were studied for mucoadhesive property and gelling temperature. They were also evaluated for in vitro cytotoxicity study in B-16 and HeLa cell lines by 3-(4,5-dimethylthiazol-2-yl)-2,5-diphenyltetrazolium bromide (MTT) assay and in vivo drug distribution study in breast tissue in healthy Wistar rats.

\section{Materials and methods}

\subsection{Materials}

Pluronic F127 $\left(M_{\mathrm{w}}=12,600\right.$, poly(ethylene oxide) (PEO) 99-poly(propylene oxide) (PPO)67-PEO99) were purchased from Sigma. Docetaxel was obtained from Panacea Biotech, Mohali, India. Glyceryl monostearate and oleic acid were purchased from CDH. Pluronic F68 as surfactant was purchased from Himedia. The HeLa and B-16 cell lines were obtained from NCCS, Pune. MTT dye was obtained from Himedia. Female rats were procured from animal house of ISF College of Pharmacy, MOGA (Punjab), India.

\subsection{Method}

\subsubsection{Preparation of nanolipid carrier}

NLC of docetaxel was prepared by emulsification and ultrasonication technique (Liu et al. 2011). Lipid and aqueous phase were prepared separately. Aqueous solution $(25 \mathrm{ml})$ was prepared by taking desired amount of surfactant, and it was dissolved in water with continuous stirring under magnetic stirring at low revolutions per minute. Lipid phase was prepared by taking desired amount of lipid (glyceryl monostearate and oleic acid) that was heated at $70{ }^{\circ} \mathrm{C}$. Docetaxel being insoluble in water was added to lipid phase and dissolved. Both aqueous phase and lipid phase were heated separately to a temperature of $60-70{ }^{\circ} \mathrm{C}$, and then aqueous phase was poured into lipid phase at the same temperature. This solution was allowed to homogenize for $3 \mathrm{~min}$ at $500 \mathrm{rpm}$, and this emulsion was immediately subjected to further size reduction using a Probe sonicator. Emulsion was probe sonicated at $60 \%$ amplitude for time $4 \mathrm{~min}$. The period of ultrasound burst was set to $9 \mathrm{~s}$ with a pause of $3 \mathrm{~s}$ between two ultrasound bursts. Obtained solution was refrigerated at $2-3{ }^{\circ} \mathrm{C}$ for $15 \mathrm{~min}$.

\subsubsection{Preparation of nanolipid carrier in gel}

Poloxamer in situ forming gels were prepared by the modified cold method (Yang et al. 2009). The required amount of pluronic F127 was slowly added to the calculated amount of NLC suspension with continuous mixing using a thermostatically controlled magnetic stirrer. The partially dissolved pluronic solutions were stored in a refrigerator and stirred periodically until clear homogenous solutions were obtained (approximately $24 \mathrm{~h}$ ).

\subsection{Optimization}

\subsubsection{Nanolipid carrier}

Different process parameters were systematically investigated to determine their effects on the particle size, polydispersity index (PDI), and zeta potential of nanolipid carriers. The process parameters included time of homogenization, time of probe sonicator, drug concentration in the solution, concentration of surfactant, and solid-to-lipid ratio.

\subsubsection{NLC in gel}

Various parameters were optimized to form NLC in gel-like pluronic F127 concentration, gelation temperature, and gelation time. 


\subsection{Characterization of nanolipid carrier}

\subsubsection{Morphology}

Transmission electron microscopy (TEM-FEI, Morgagni $268 \mathrm{D}$, the Netherland) was employed for particle imaging for visualization of shape and morphology of the prepared nanolipid carriers. A drop of the sample was placed onto a carbon-coated copper grid to leave a thin film on the grid. The film was negatively stained with $1 \%$ phosphotungstic acid solution and dried. A drop of the staining solutions was added on to the film, and the excess of the solution was drained off. The grid was allowed to dry at ambient temperature, and samples were viewed under TEM and photographs were taken at suitable magnification.

\subsubsection{Particle size and distribution}

Size and size distribution (PDI) of optimized formulation was determined by laser diffractometry using Beckman Coulter Delsa $^{\mathrm{TM}}$ Nano C Particle Analyzer. For the measurement of size and PDI, $2 \mathrm{ml}$ of nanolipid carrier was placed into cuvettes of Beckman coulter, and measurements were recorded. Size and PDI of NLC containing docetaxel were determined.

\subsubsection{Charge on the particle}

Particle charge determines physical stability of nanolipid carrier. Particle charge is quantified as zeta potential, which is measured via electrophoretic mobility of particles in an electrical field. The colloids with high zeta potential (negative or positive) are electrically stabilized and with low zeta potential tends to coagulate. Zeta potential was measured using Beckman Coulter Delsa ${ }^{\mathrm{TM}}$ Nano C Zeta Sizer.

\subsubsection{Differential scanning calorimetry}

Differential scanning calorimetry (DSC) is a thermoanalytical technique which is used to study degree of crystallinity and polymorphic transitions or thermal transitions involving energy changes during the process of formulation. The phase transition of docetaxel nanolipid carrier was analyzed by differential scanning calorimetry (DSC model, TA instruments, Q-20, USA) in a perforated aluminum sealed 50- $\mu \mathrm{l}$ pan at a heating rate of $10^{\circ} \mathrm{C} / \mathrm{min}$ from 30 to $300{ }^{\circ} \mathrm{C}$ using nitrogen as blanket gas $(20 \mathrm{ml} / \mathrm{min})$.

\subsubsection{Entrapment efficiency}

Percent drug entrapment was determined and expressed as the ratio of experimentally measured amount of drug in dispersion and initial amount used for entrapment. Two milliliters of suspension (undiluted) was dropwise applied on the top of the gel bed of the Sephadex minicolumn. Columns were centrifuged in centrifuge (R-24C, Remi Instruments, India) at 3,000 rpm for $3 \mathrm{~min}$ to remove unentrapped drug. Elute was removed and $2 \mathrm{ml}$ saline was applied to each column and centrifuged as previously mentioned. Amount of drug entrapped in the nanolipid carriers was then determined by disrupting the vesicles by adding SLS $(0.3 \%$, $w / v)$ in phosphate-buffered saline (PBS) pH 7.4 and heating for $20 \mathrm{~min}$ and bringing it to room temperature which was centrifuged at 10,000 rpm for $10 \mathrm{~min}$ and assayed for drug content by UV spectrophotometer at $231 \mathrm{~nm}$.

The percentage drug entrapment was measured as:

$\% \mathrm{DEE}=\frac{\text { Amount of drug entrapped }}{\text { Amount of drug added }} \times 100$

The individual values for triplicate determinations and their mean values were reported.

\subsection{In vitro characterization of gel}

Optimized formulation was characterized for various parameters like rheological studies, mucoadhesive strength, and in vitro drug release.

\subsubsection{Rheological studies}

Rheological behaviors of the gel were investigated by studying their viscosity as functions of temperature in Brookfield $\mathrm{R} / \mathrm{S}$ plus Rheometer CPS were employed to determine the rheological properties of NLC in gel systems using CPS 25 spindle equipped with a cone and plate test geometry. The viscosity of each sample was determined before and after gel formation by adjusting the temperature of the sample holder. Since the pluronic F127 gel (20\% solution) in the range of 32 to $34{ }^{\circ} \mathrm{C}$, estimation was conducted at 30 and $35^{\circ} \mathrm{C}$. Each point is the mean of three readings.

\subsubsection{Mucoadhesive strength}

Mucoadhesive forces of all the prepared formulations were determined using a mucoadhesive force measuring device, which is a modified balance. This balance was developed in our laboratory with slight modifications, according to the previously reported methods. The mucoadhesive force of the formulations under examination was determined by measuring the force required to detach the formulation from breast tissue detached from Wistar rats attached to one side using the measuring device. At the right arm of the balance, breast tissue was horizontally glued to the lower surface of the right pan of the modified balance. The tissue was attached to one side and was hydrated with distilled water 
prior to the mucoadhesion test. One milliliter of formulation was placed on the upper surface of slide.

The upper stage of the modified balance containing the hydrated breast tissue of Wistar rats was adjusted to be in contact with the preparation. The water was allowed to drip from the infusion set into a preweighed plastic jar placed on the left pan of the balance at a constant rate. The dripping of water was stopped when the tissue was detached from the tested sample, the filled plastic jar was reweighed, and the mass of water required to detach the tested sample from the tissue was calculated from the difference. Measurements were repeated three times for each of the gel preparations, but a fresh tissue and smooth gel surface was created before each measurement. The weight required for the detachment was calculated, and mucoadhesive strength was determined according to the formula given below.

Mucoadhesive Strength $=$ Force of Adhesion/Area

wherein Force of Adhesion $=(\mathrm{m}(\mathrm{g}) \mathrm{g}($ accdueto gravity $) /$ 1,000 .

\subsection{In vitro drug release}

\subsubsection{In vitro drug release from nanolipid carrier}

In vitro drug release studies were performed by dialysis bag method using shaking incubator (LSB-1005 RE Daihan Labtech Co. Ltd., Korea) at rotation speed of $100 \mathrm{rpm}$. SLS $(0.3 \%, w / v)$ phosphate buffer ( $\mathrm{pH} 7.4)$ was used as dissolution medium. Each dialysis bag (pore size $12 \mathrm{kDa}$, Sigma Chemical Co., USA) was loaded with nanolipid carrier equivalent to $800 \mu \mathrm{g}$ of drug. Volume and temperature of dissolution medium were $100 \mathrm{ml}$ and $37.0 \pm 0.2{ }^{\circ} \mathrm{C}$, respectively. At predetermined time interval, samples $(3 \mathrm{ml})$ were withdrawn, replaced with same volume of fresh media, filtered, and assayed for drug content at $231 \mathrm{~nm}$ against blank using UVvisible spectrophotometer. Mean results of triplicate measurements and standard deviation were reported.

\subsubsection{In vitro drug release from nanolipid carrier in gel}

Membrane-less diffusion system was used for studying the release of docetaxel from pluronic F127 gel as reported previously. A volume of 2-ml pluronic 127 gel was put in a flat-bottomed vial (internal diameter about $18 \mathrm{~mm}$ ) followed by adding $8 \mathrm{ml}$ physiological saline $(0.9 \% \mathrm{NaCl}$, $w / w)$ gently on the surface of gels, and then the vial was shaken in a thermostatic shaker (LSB-1005 RE Daihan Labtech Co. Ltd., Korea) under $37{ }^{\circ} \mathrm{C}$ at $40 \pm 10 \mathrm{rpm}$. Aliquot of $2 \mathrm{ml}$ sample of the supernatant solution was withdrawn at predetermined.

\subsection{Stability studies}

Selected and optimized formulations of NLC-DTX were subjected to stability testing for 30 days in a desiccator at room temperature. The samples were observed at the predetermined time intervals of 1,10 , and 30 days and evaluated for appearance, particle size, and PDI.

2.8 In vitro anticancer activity studies in cell lines

\subsubsection{In vitro cytotoxicity studies (MTT assay)}

Cell culture establishment and maintenance HeLa and B-16 cell lines obtained from NCCS Pune were maintained in desired media supplemented with $10 \%$ inactivated fetal bovine serum, $100 \mathrm{U} / \mathrm{ml}$ penicillin, and $100 \mu \mathrm{l} / \mathrm{ml}$ streptomycin incubated at $37{ }^{\circ} \mathrm{C}$ and $5 \% \mathrm{CO}_{2}$ in humidifier incubator. After attaining $80 \%$ confluence cells were subcultured by trypsinization with $0.25 \%$ trypsin solution under sterile conditions.

MTT assay The cytotoxicity of various drug solutions was determined by tetrazolium-based colorimetric assay (MTT assay). Cells were plated in 96 -well plates at $5 \times 10^{3}$ per $100 \mu \mathrm{l}$ per well with density determined on the basis of growth characteristics of each cell line. After overnight incubation, triplicate wells were treated with varying concentration of compounds ranging from 0.01 to $50 \mu \mathrm{M} / \mathrm{ml}$ and standard docetaxel incubated for 3 days. After 3 days, medium was replaced with $2 \mu \mathrm{l}$ of MTT solution $(5 \mathrm{mg} / \mathrm{ml})$, and cells were incubated for $3 \mathrm{~h}$. Formazan crystals were dissolved in dimethyl sulfoxide (DMSO). The relative percentage of metabolically active cells compared with untreated controls was then determined on the basis of mitochondrial conversion of MTT to formazan crystals that were dissolved in DMSO. Formazan crystals were dissolved in DMSO. Spectrophotometric absorbance of sample was determined by microplate reader (Biorad) at 570/630 $\mathrm{nm}$. Concentrations of samples showing $50 \%$ reduction in cell viability (i.e., $\mathrm{IC}_{50}$ values) were then calculated.

\subsubsection{Mechanism of cell death (apoptosis) study}

Acridine orangelethidium bromide assay Cancer cells grown in six-well plate $(12,000$ cells/well) were treated with and without drug for $24 \mathrm{~h}$. B-16 cells were pelleted by centrifuged at 5,000 rpm for 5 min using a Beckman Model TJ-6 centrifuge and washed with $1 \mathrm{ml}$ of cold PBS once. Cell pellets were then re-suspended in 25-ml cold PBS, and

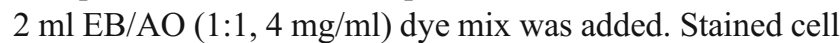
suspensions $(10 \mathrm{ml})$ were placed on a clean microscope slide and covered with a cover slip. Cells were viewed and counted using inverted microscope (Olympus CKX 41) at $\times 400$ magnification. Pictures were taken with a Sony color 


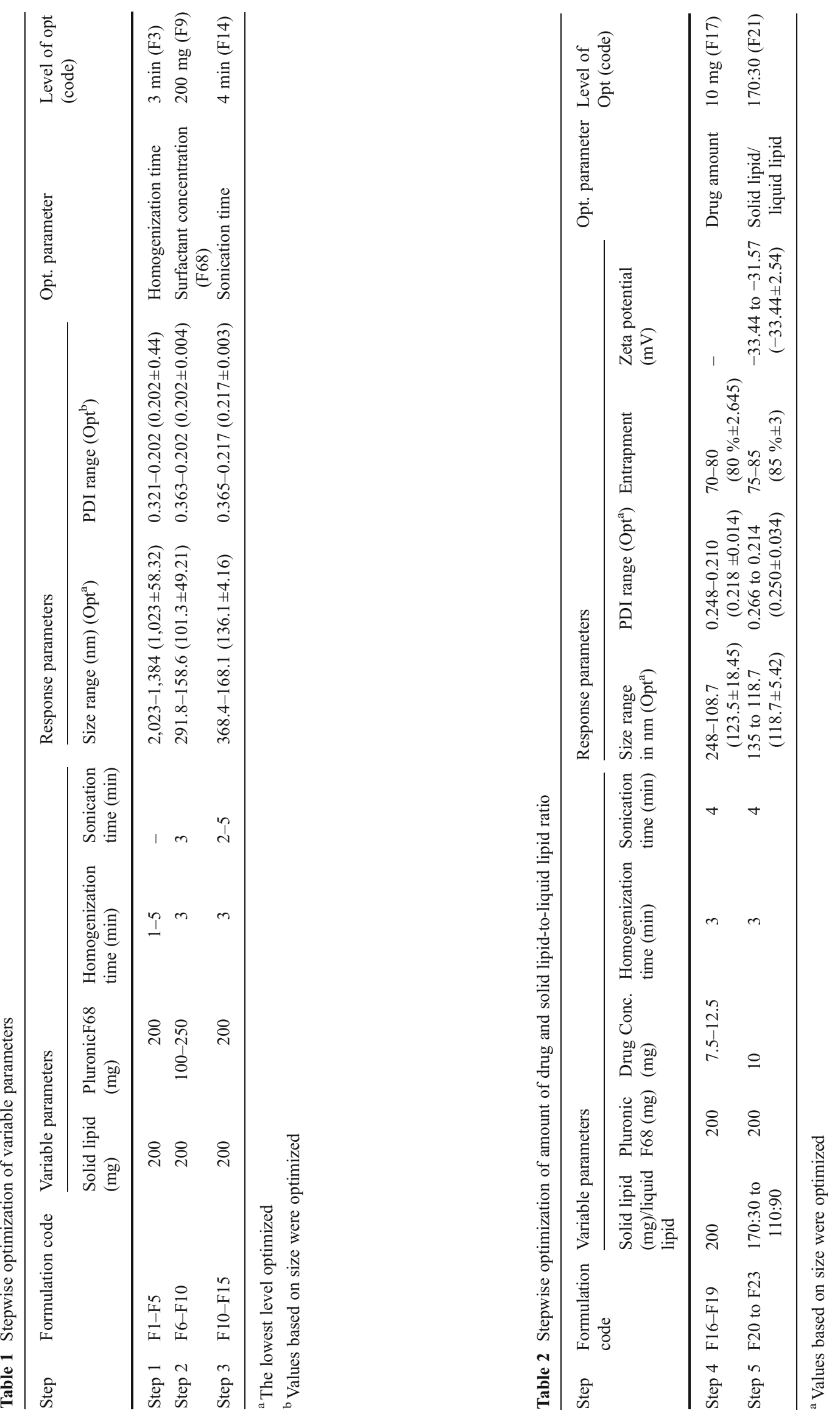


video camera. Tests were done in triplicate, counting a minimum of 100 total cells each. The differential uptake of these two dyes allows the identification of apoptotic and non-apoptotic cells (Babykutty et al. 2009).

\subsection{In vivo breast tissue homogenate studies}

For tissue distribution studies, group of rats $(n=4)$ were administered with prepared nanolipid carriers at a dose of $200 \mu \mathrm{g} / \mathrm{kg}$. After administration of docetaxel nanolipid carrier, tissue was collected at various sampling points to determine the percent drug recovery at different time interval.

Percentage drug recovery from tissue was obtained by administration of pure drug followed by administration of prepared nanolipid carrier and nanolipid carrier in gel through intralesional route. Three groups of rats were taken for tissue distribution studies. Each group had four rats at each sampling point. The formulation was administered intralesionally with a syringe.

\subsection{Statistical analysis}

All the experiments were carried out three times independently. The data obtained were expressed in terms of "mean \pm standard deviation" values. These values were analyzed using two-way ANOVA.

\section{Results}

\subsection{Optimization}

\subsubsection{Nanolipid carrier}

The parameters such as homogenization time, sonication time, weight ratio of drug to lipids (glyceryl monostearate and oleic acid), weight ratio of solid lipids to liquid lipids, and the concentration of pluronic F127 were optimized each at various levels taking the particle size, PDI, zeta potential,
Table 3 Optimization parameters of gel

\begin{tabular}{llcc}
\hline S.No. & $\begin{array}{l}\text { Polymer concentration } \\
(\%)\end{array}$ & $\begin{array}{l}\text { Gelation } \\
\text { temperature }\end{array}$ & Gelation time \\
\hline 1 & 16 & $36-38$ & 60 \\
2 & 18 & $34-36$ & 45 \\
3 & 20 & $30-32$ & 45 \\
4 & 22 & $27-29$ & 30 \\
5 & 24 & $24-26$ & 30 \\
\hline
\end{tabular}

and entrapment efficiency as response parameter. When one factor was under investigated, the other three were fixed (Tables 1 and 2).

Optimized formulation (F14) with 3 min homogenization time, 4 min sonication time, $200 \mathrm{mg}$ pluronic F68 as surfactant, and $10 \mathrm{mg}$ as drug amount was further optimized for solid-to-lipid ratio keeping all the above-optimized parameters constant at various levels. Particle size and drug entrapment efficiency of NLCs prepared with various amounts of liquid lipid are given in Fig. 1.

\subsubsection{Nanolipid carrier in gel}

Various parameters were optimized like polymer concentration which affects the diffusivity of drug in higher polymer concentration (like $20 \%, 22 \%(w / w)$ ) in formulation of pluronic F127 gel. It was observed that the diffusion coefficients tend to decrease as the polymer content increases which were also reported for pluronic F127. Polymer concentration also affected gelation temperature and gelation time (Table 3).

The performance criteria for gel depend on physiological temperature of breast tissue which was optimized to $30-32{ }^{\circ} \mathrm{C}$. The optimum time required by the formulation which underwent sol-gel transition was $45 \mathrm{~s}$, which was optimum for the gel to easily get attached to tissue. Thermoreversible gel enhances the bioavailability of drug at breast tissue by
Fig. 1 Optimization of solid lipid to liquid lipid

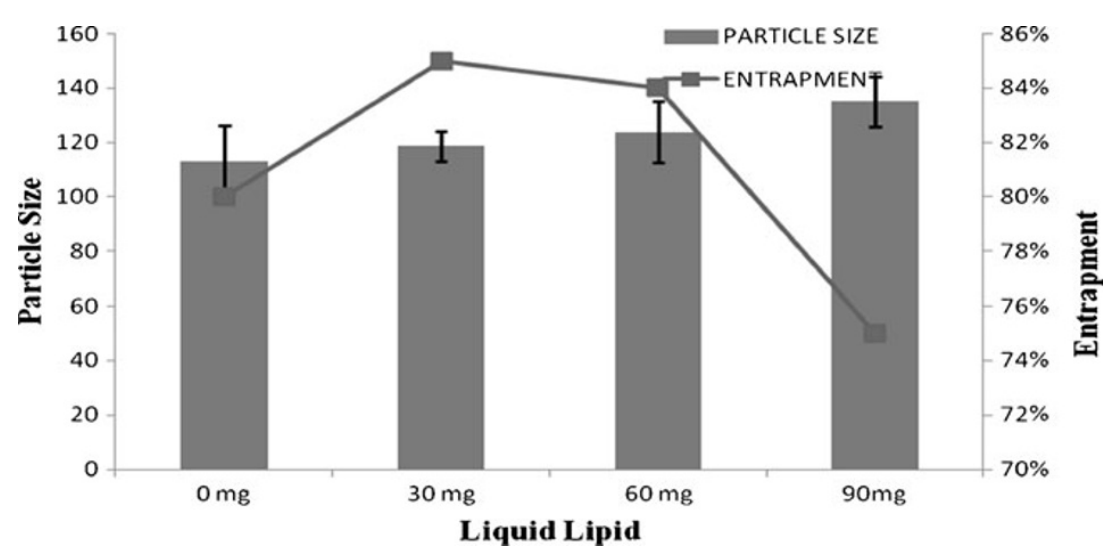


Fig. 2 TEM photograph of nanolipid carrier of docetaxel
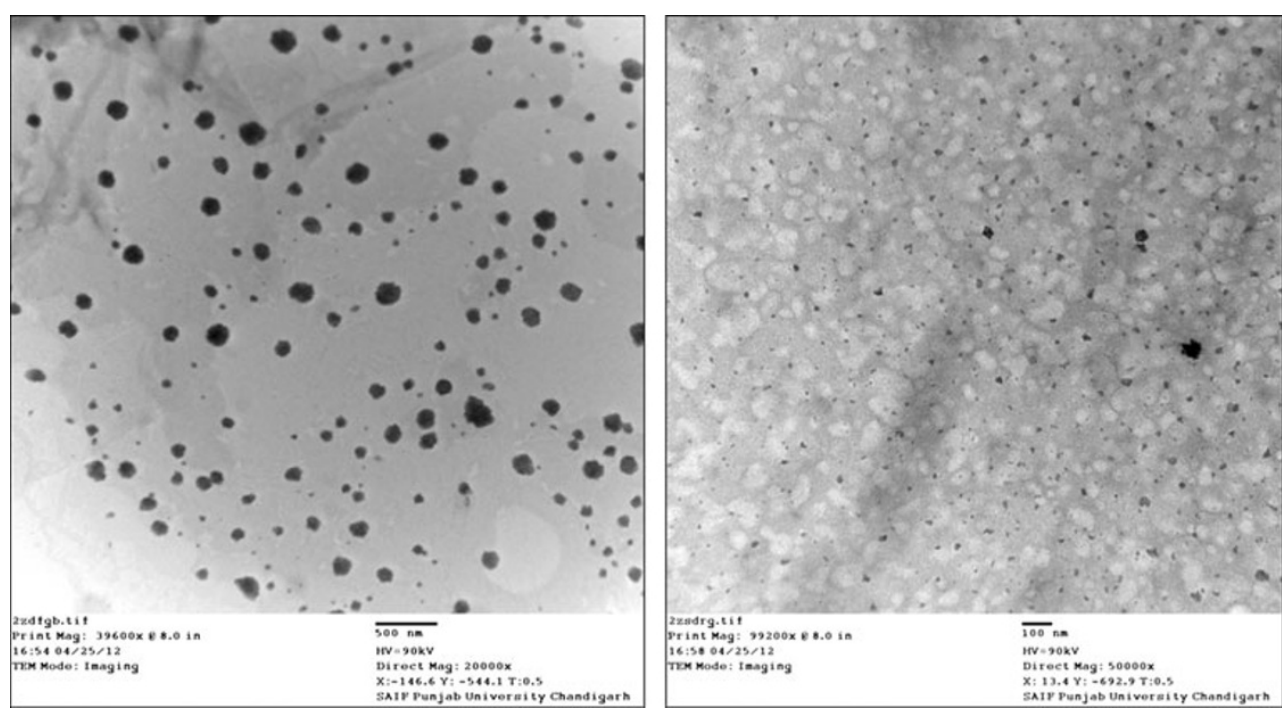

immobilizing the formulation at the site so that drug is released and absorbed for a longer duration of time

\subsection{Characterization of nanolipid carrier}

In vitro characterization included parameters like morphology, size and size distribution, particle charge, DSC, entrapment efficiency, and in vitro drug release.

\subsubsection{Morphology}

Optimized formulation was visualized under TEM for surface morphology (Liu et al. 2011. TEM photographs revealed that nanolipid carriers are spherical in shape. TEM images of nanolipid carrier of docetaxel are shown in Fig. 2.

\subsubsection{Particle size and distribution}

Size and size distribution of optimized nanolipid carrier of docetaxel was determined by photon correlation spectroscopy

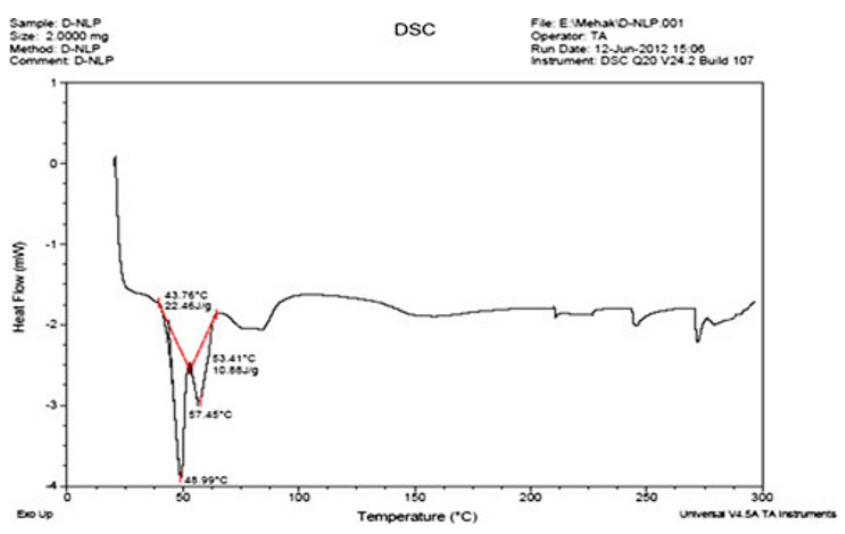

Fig. 3 DSC of nanolipid carrier method using zetasizer (Bechman Coulter), and the results are tabulated in Tables 1 and 2. Size and size distribution of optimized formulation was found to be $123.5 \mathrm{~nm}$ with PDI of 0.218 .

\subsubsection{Particle charge}

Charge on the particles is expressed in terms of zeta potential, and its determination is useful to evaluate physical stability of a nanolipid carrier. Zeta potential values determined for various batches of nanolipid carrier are tabulated in Table 2. Zeta potential of the optimized batch (F21) of the nanolipid carrier was $-33.44 \pm 2.54 \mathrm{mV}$.

\subsubsection{Differential scanning calorimetry}

The mixing behavior of solid lipid and lipophilic solubilizer in the NLC was investigated by DSC (Bunjes et al. 1996; Hou et al. 2003). It gave information about the melting, crystallization behavior, polymorphism, and crystal ordering of the solid and liquid constituents of the nanolipid carriers. The melting endotherm of NLC (Fig. 3) showed complete absence of docetaxel peak, indicating that docetaxel was completely solubilized inside the lipid matrix of NLC. DSC thermogram of

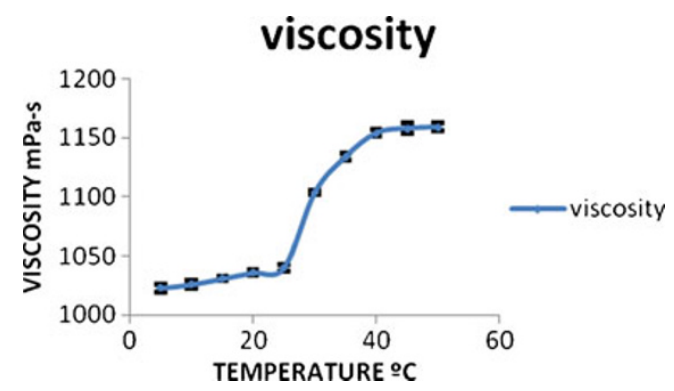

Fig. 4 Graph explaining the rheological behavior of gel 
Table 4 Optimization of force of adhesion and mucoadhesive force

\begin{tabular}{llll}
\hline S.No. & Polymer concentration (\%) & Force of adhesion (dyne) & Mucoadhesive force $\left(\right.$ dyne $\left./ \mathrm{cm}^{2}\right)$ \\
\hline 1 & 16 & $28.23 \pm 0.65$ & $2.51 \pm 0.08$ \\
2 & 18 & $32.39 \pm 0.95$ & $2.9 \pm 0.215$ \\
3 & 20 & $36.09 \pm 0.73$ & $3.07 \pm 0.175$ \\
4 & 22 & $39.33 \pm 0.27$ & $3.4 \pm 0.270$ \\
\hline
\end{tabular}

NLC showed endothermic with lower onset temperature $\left(49.92^{\circ} \mathrm{C}\right)$ and peak temperature $\left(53.37^{\circ} \mathrm{C}\right)$ values from those of the bulk lipid material (Pople and Singh 2011).

\subsubsection{Entrapment efficiency}

Entrapment efficiency of docetaxel nanolipid carrier was estimated and repeated three times. All the formulations showed good reproducible results (Table 2). Entrapment efficiency of docetaxel was found to be $85 \% \pm 3$ confirming good entrapment efficiency of nanolipid carrier. Most solid lipids form highly crystalline particles, with a perfect lattice leading to drug expulsion. On the other hand, the imperfection (lattice defects) of the lipid structure could offer space to accommodate the drug. Modification of lipid by incorporation of lipophilic solubilizer molecules might have resulted in the formation of less perfect crystals with many imperfections offering space to accommodate the dissolved drug. The minimum amount of solubilizer used (just required to dissolve the drug) might be responsible for complete immobilization of liquid nanodroplets of drug in the crystal imperfections of solid lipid avoiding ejection of the liquid lipid during the crystallization process at the cooling step (Westesen et al. 1997).

\subsection{Characterization of gel}

\subsubsection{Rheological studies}

Rheological studies revealed that the viscosity of the NLCs remained almost constant up to $30{ }^{\circ} \mathrm{C}$ followed by sudden rise in viscosity up to $37^{\circ} \mathrm{C}$. Beyond this temperature, no rise in viscosity is observed (Fig. 4). This indicates that the phase transition from sol to gel starts at $30^{\circ} \mathrm{C}$ and complete conversion to viscous gel would take place at $37^{\circ} \mathrm{C}$. This is indicated by no rise in viscosity once the gel is completely formed.

\subsubsection{Mucoadhesive strength}

Good mucoadhesive behavior is a prerequisite for a gel to exhibit long residence time for effective and complete absorption and sustained action. This property is determined in terms of force of adhesion in dynes or mucoadhesive force in dynes per square centimeter. The $20 \%$ pluronic gel showed force of adhesion of $36.09 \pm 0.73$ dyne and mucoadhesive force $3.07 \pm 0.175 \mathrm{dyne} / \mathrm{cm}^{2}$ (Table 4).

\section{In vitro drug release}

Cumulative release of docetaxel from PF127 gel at $37{ }^{\circ} \mathrm{C}$ was displayed in Fig. 5. It was obvious that docetaxel released much slower from docetaxel-NLC in Gel than from plain drug suspension of docetaxel and NLC of docetaxel. In the first $16 \mathrm{~h}$, about $28 \%$ of DTX was released from DTX-NLC and DTX-NLC in gel formulation against $96.14 \%$ of DTX form plain drug suspension. Thereafter, release rate of DTX from DTX-NLC formulation was faster up to $35 \mathrm{~h}$ when the maximum drug release (96\%) was reached while in case of DTX-NLC in gel, the same rate of drug release was continued up to the
Fig. 5 Comparison of cumulative drug release of DTX from NLC in gel, NLC, and plain drug suspension

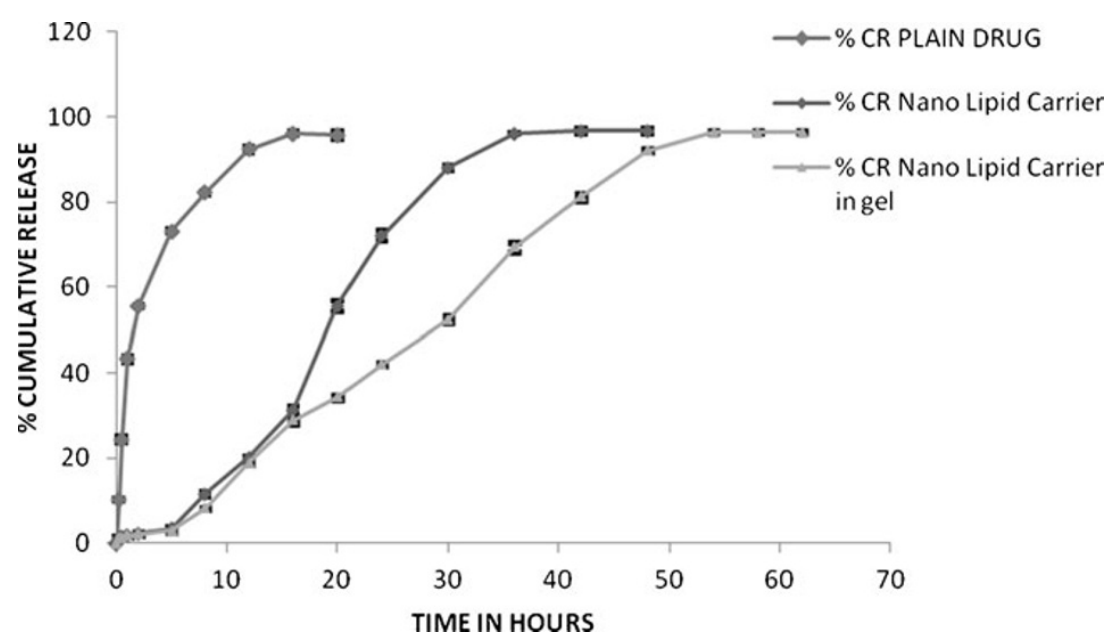


Fig. 6 Average particle size and PDI of nanolipid carrier during stability study

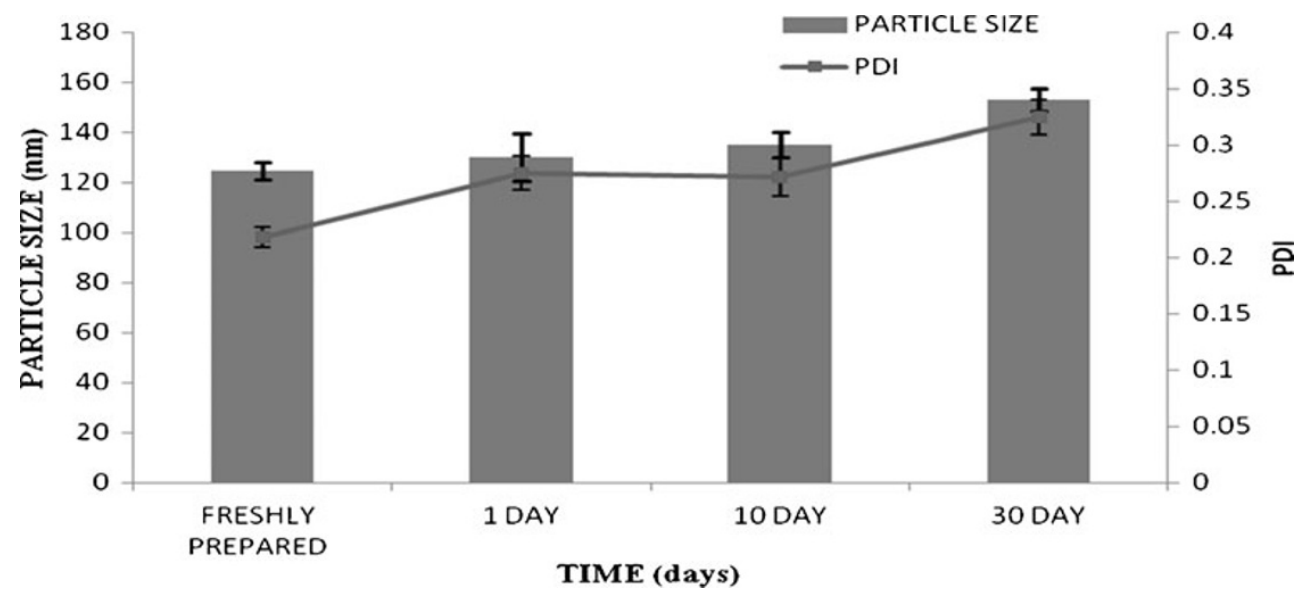

maximum of $96 \%$ in about $53 \mathrm{~h}$. These results clearly show that the maximum drug release in all three formulations was $96 \%$ of the total drug but rate and style of release was different. Plain drug suspension showed initial burst release of 45 to $50 \%$ drug in about $1.5 \mathrm{~h}$ followed by slower release up to the maximum of $96 \%$ in $16 \mathrm{~h}$. Both DTX-NLC and DTX-NLC in gel formulations showed continuous release up to $96 \%$ in 35 and $53 \mathrm{~h}$, respectively. However, the release was quite faster in case of DTX-NLC than from DTX-NLC in gel formulation. The difference between the release properties of DTX from plain drug suspension and DTX-NLC and DTX-NLC in gel is evidently attributed to the prolonged release function of NLC in gel. Docetaxel is present in dissolved state in NLC, and the release is possible into aqueous media by partitioning which would be obviously very slow. In addition, release of docetaxel from the drug-loaded NLC in gel has to partition from NLC into the gel media in the first phase and then transfer to the aqueous dissolution media by partitioning and diffusion across the viscous gel. The release kinetic of docetaxel from DTX-NLC in gel showed linear curve $\left(r^{2}=0.997\right)$ when root of time was plotted against cumulative drug release (Higuchi model) indicating which indicated that the drug released by dissolution and diffusion.

\section{Stability studies (Souza et al. 2011)}

The results of stability studies indicated no change in particle size and PDI of formulation till 10 days, but there was slight increase in particle size from 123.5 to $153.2 \mathrm{~nm}$ in 30th day sample. However, the formulation was clear up to 30 days (Fig. 6).

\section{In vitro anti cancer activity in cell line}

\subsection{Cytotoxicity studies (MTT assay)}

\subsubsection{Dose-dependent studies}

The comparative cytotoxicity studies of different formulations like plain drug suspension, docetaxel nanolipid carrier, and nanolipid carrier in GEL were done using MTT assay. Percentage cell viability of all formulations at different concentrations in HeLa cell lines and B-16 cell line was evaluated and presented in Fig. 7. NLC of docetaxel showed a clear dose-dependent cytotoxicity against these cells with DTX at an equivalent dose from 0.01 to $50 \mu \mathrm{M} / \mathrm{ml}$. The $\mathrm{IC}_{50}$ values calculated for docetaxel plain drug suspension, NLCDTX, and NLC-DTX in gel was shown in Table 5.
Fig. 7 Comparison of MTT assay of formulations on HeLa cell lines (a) and B-16 cell line (b)

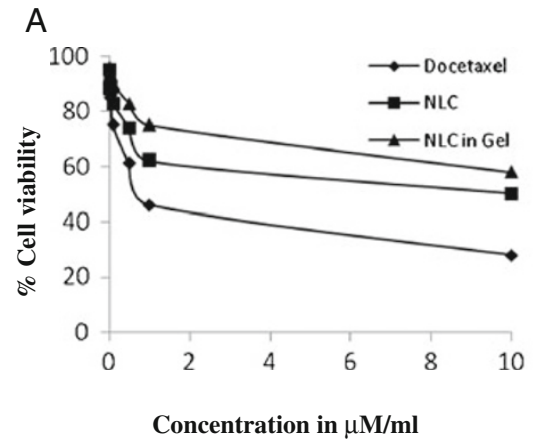

Concentration in $\mu \mathrm{M} / \mathrm{ml}$

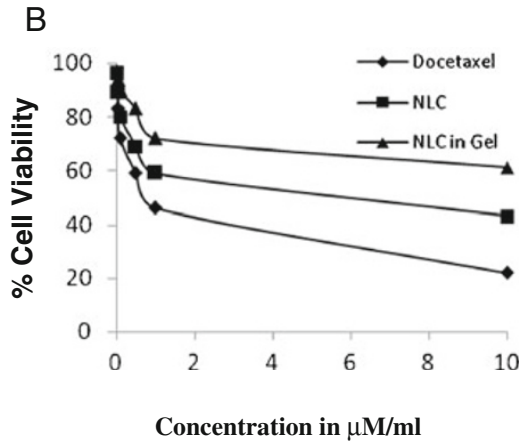


Table $5 \mathrm{IC}_{50}$ value of formulation

\begin{tabular}{lll}
\hline Formulation & $\mathrm{IC}_{50}$ value $\mathrm{B} 16(\mu \mathrm{M})$ & $\mathrm{IC}_{50}$ value $\mathrm{HeLa}(\mu \mathrm{M})$ \\
\hline Plain drug suspension & $0.88 \pm 1.03$ & $0.92 \pm 1.21$ \\
NLC & $8.97 \pm 2.09$ & $9.2 \pm 1.99$ \\
NLC in gel & $11.96 \pm 1.51$ & $11.68 \pm 2.21$ \\
\hline
\end{tabular}

\subsubsection{Time-dependent cell viability studies}

The comparative cytotoxicity studies of different formulations like plain dug suspension, docetaxel nanolipid carrier, and nanolipid carrier in GEL were done using MTT assay. Percentage cell viability of all formulations at different concentrations in HeLa cell lines was evaluated after exposure for varied time duration $(24,48,72$, and $96 \mathrm{~h})$ and is presented in Fig. 8.

\subsection{Cell death [apoptosis] studies}

The induction of nanolipid carriers mediated cell apoptosis was observed by acridine orange/ethidium bromide double staining of treated cell nuclei with optimized dose of nanocarrier lipid. Microscopic images of the dual stained cells, presented in Fig. 9, show that the live cell nuclei stained green due to acridine orange uptake and their numbers gradually decreased with time owing to more cell death figure, which correlates with the TEM images. One can observe progressive nuclear uptake of ethidium bromide due to cell membrane perforation during apoptosis, which stained nuclei red, and such an effect was prominent in $24 \mathrm{~h}$. Microscopic images of treated as well as untreated cells at $24 \mathrm{~h}$ indicate that live untreated cells had well-organized chromatin structures, whereas the treated cells had fragmented or condensed chromatin consisting of apoptotic nuclei. Therefore, the nuclear staining experiment shows that apoptosis was seen in $24 \mathrm{~h}$ after addition of nanolipid carrier to the culture medium.

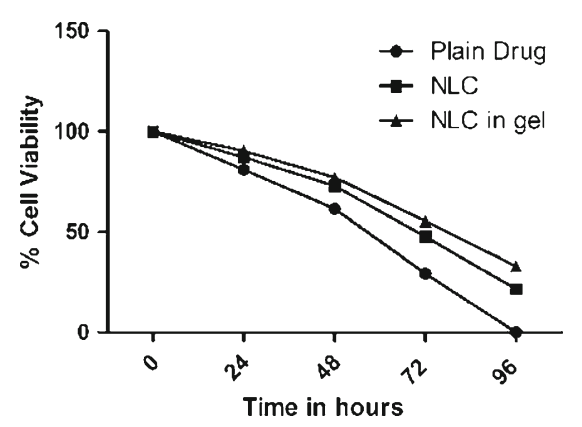

Fig. 8 Percentage cell viability after exposure with different formulations for varied time duration on HeLa cell line

\section{In vivo breast homogenate studies}

Tissue homogenate studies were performed to determine the residence time of docetaxel after local intralesional administration of the drug into breast tissue as plain drug suspension in water, DTX-NLC, and DTX-NLC in gel. Drug concentration estimated in breast homogenate samples at definite time intervals is shown in Fig. 10. The results indicated maximum residence time of the formulation and drug concentration in case of DTX-NLC in gel followed by DTX-NLC and then plain drug suspension. The drug released from DTX-NLC in gel took prolonged time $(60 \mathrm{~h})$ to get almost cleared from the breast tissue while it was 36 and $24 \mathrm{~h}$ in case of DTX-NLC and plain drug suspension, respectively. Thus, the study revealed that enough amount of drug was reaching into retained in the effector organ for long time in a controlled manner (Fig. 10).

\section{Statistical analysis}

All the experiments were carried out three times independently. The data obtained were expressed in terms of "mean \pm standard deviation" values. These values were analyzed using two-way ANOVA, and results were found to be significant with $p<0.001$.

\section{Discussions}

Investigations have been made on other delivery approaches for various gynecological cancers like breast cancer which have widely spread in world among females. Docetaxel is a potent anti-cancer drug used in treatment of breast cancer which possesses many unavoidable side effects, and various approaches have been developed with intravenous infusion or I.V. bolus to produce sustain effect with limited success. The present investigation is focused on the local delivery of drug into the tumor as a better method in administrating the drug. The work performed in this present study has also used novel approaches of using docetaxel-loaded NLC and DTX-NLC in pluronic gel to improve the residence time of the formulation in the effector organ after intralesional injection into breast tissues. The structural integrity of NLC containing liquid and solid lipid core could increase the solubility of docetaxel; the optimal zeta potential and particle size of NLC could keep docetaxel stably entrapped in inner core of NLC (Hu et al. 2006). This system was incorporated into pluronics 127 which is composed of an outer shell of hydrophilic PEO and a hydrophobic PPO core, which possess thermoreversible property which is in sol form at low temperature and gets converted into gel at physiological temperature. 

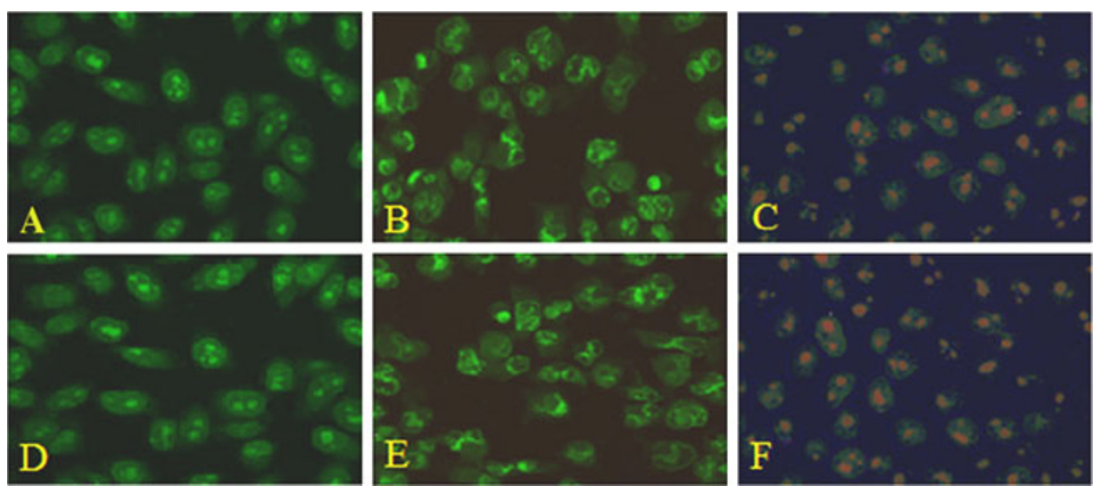

Fig. 9 Fluorescence micrographs (magnification $\times 400$ ) of acridine/ ethidium bromide stained B-16 mouse melanoma cell line. a, d Unexposed (control cells have normal green nucleus indicating live cells). b, e Cells exposed to $\operatorname{IC}_{50}(0.88$ and $11.96 \mu \mathrm{g} / \mathrm{ml})$ of plain drug and NLC

The difference between the release properties of docetaxel from plain drug suspension, NLC of docetaxel, and NLC in gel is evidently attributed to the prolonged release function of NLC in gel. Docetaxel being lipophilic in nature was held by the lipid core of the NLC and the drug released mainly through dissolution and diffusion. This result implied that docetaxel could be released slowly from NLC of docetaxel and could keep constant concentration for relatively long period. Therefore, NLC of docetaxel in gel might reduce frequent administration of drug which was desired clinical application of this project.

NLC of docetaxel and the NLC in gel showed decreased cytotoxicity than free docetaxel against both B-16 and HeLa cell lines. However, Paola et al. (2012) reported that the antiproliferative effect of docetaxel appeared much earlier when the drug was encapsulated in lipid nanoparticles than when it was free when subjected to flow cytometric studies with breast carcinoma (MCF-7) cells. In addition, Xu et al. (2009) reported increased anti-tumor activity of docetaxel-loaded hepatoma- in gel, respectively, for $24 \mathrm{~h}$ bright green nucleus with condensed or fragmented chromatin suggesting apoptosis. c, f Cells exposed to $\mathrm{IC}_{50}$ $(0.88$ and $11.96 \mu \mathrm{g} / \mathrm{ml})$ of plain drug and NLC in gel, respectively, for $24 \mathrm{~h}$ have a structurally normally orange nucleus indicate necrosis

targeted solid lipid nanoparticle (SLN) prepared with galactosylated dioleoylphosphatidyl ethanolamine in comparison to taxotere or docetaxel-loaded nontargeted SLN following IV injection. In most of these experiments, it is clear that the increased activity of docetaxel-loaded lipid nanoparticles is by increased uptake of these particulates by enhanced permeation and retention mechanism and may also due to avoidance of efflux process. Till today, no reports are available to authenticate the uptake of intralesionally injected lipid nanoparticles by tumor tissue intact. Hence, the advantage of intralesional injection is due to avoidance of systemic side effect and improved therapeutic efficacy as a result of sustained release of the anticancer agent loaded in lipid particles or in gel system. Decreased anticancer activity of docetaxel-loaded NLC and the NLC-loaded gel in comparison to docetaxel as observed in our experiments is also due to slow rate of uptake of nanolipid carriers, and the docetaxel release from these NLCs is the critical factor for the drug uptake by cells. Colloidal delivery systems like NLCs administered by intramammary injection
Fig. 10 Percent drug recovered in breast tissue

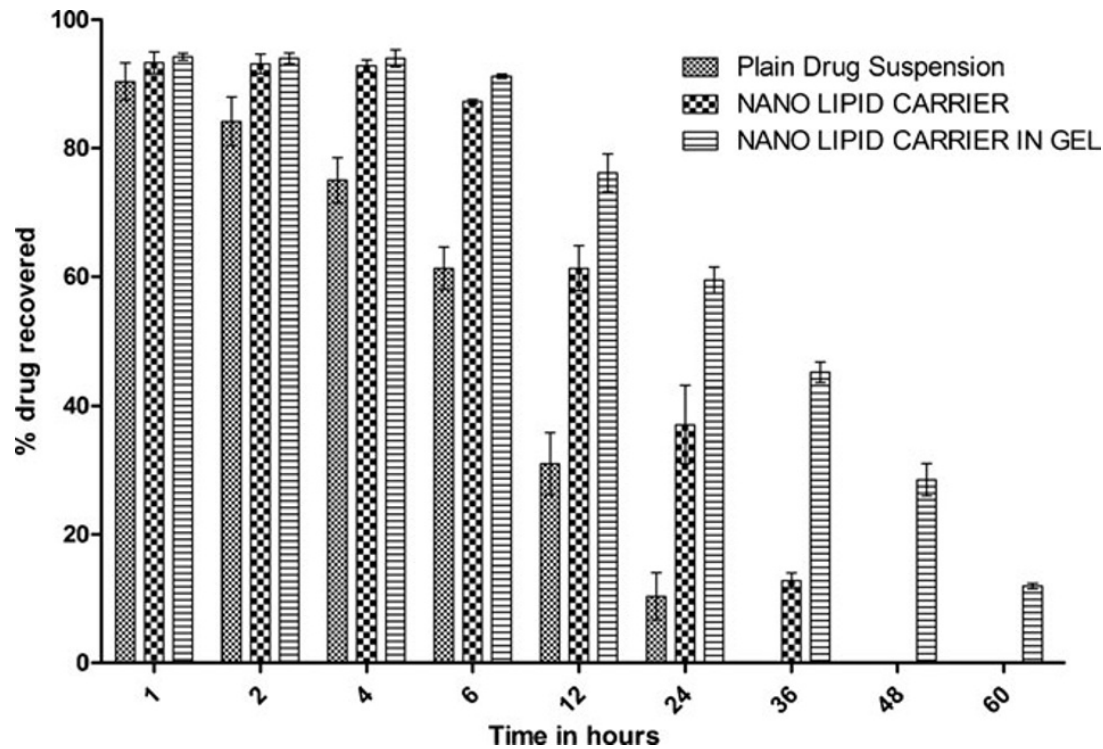


could yield high local concentration in the breast tissue and its associated lymph drainage area (Berg and Christophersen 1956). Similar conclusions were also drawn by Mendes et al. (2009) based on their work on the uptake by breast carcinoma and lymph nodes of a lipidic nanoemulsion after intralesional injection into the patients. Luciano et al. (1995) also reported that the potentiation of the antitumor actions of IFNs administered by intramammary injection to human breast cancer xenografts growing bilaterally in nude mice was effective at the local but not at the systemic level indicating that the drug accumulation in the local area of breast tissue is a critical factor in breast tumor regression.

In vivo breast tissue homogenate studies were performed to determine the local drug concentration reaching into targeted organ through increased residence time due to slow release of the drug from the DTX-NLC and DTX-NLC in gel formulations. In vivo results matched with the release pattern of in vitro release profile of the drug. The results revealed that enough amount of drug released was retained in the organ in a controlled manner with slow elimination from the body. The organ distribution study confirmed that drug released from the gel was in a controlled manner which meets the desired application of nanolipid carrier in gel.

\section{Conclusions}

In the present study, we first developed a NLC system which was incorporated into pluronic 127 gel which possesses thermosensitive property. In addition, its capacity to trap poorly soluble drug in NLC was insensitive to temperature. According to our study data, the release of docetaxel from NLC in gel in vitro and in vivo was sustained for more than $60 \mathrm{~h}$. The NLC in gels can solubilize and stabilize DTX. However, anti-tumor activities of docetaxel-loaded NLC and the NLC in gel were lower than plain docetaxel when tested on B-16 and HeLa cell lines in vitro. Specific administration, such as intralesional delivery, likewise improved the local drug concentration around tumors and could enhance the therapeutic effects of NLC in gel against breast tumor. As a consequence, local administrations of docetaxel in NLC system appeared to be good physical targeting approaches for solid tumor treatment. At the same time, they provide scientific basis for further studies regarding the application of thermosensitive gel loaded with waterinsoluble drugs in other clinical cancer therapy studies.

\section{References}

Babykutty S, Padikkala J et al (2009) Apoptosis induction of Centella asiatica on human breast cancer cells. African Journal of Traditional, Complimentary and Alternative Medicines 6(1):9-16
Berg FH, Christophersen MW (1956) An experimental study of intramammary injection of $\mathrm{Au}^{198}$. Ann Surg 143(1):92-97

Bunjes H, Westesen K et al (1996) Crystallization tendency and polymorphic transitions in triglyceride nanoparticles. Int J Pharm 129:159-173

Hou D, Xie C et al (2003) The production and characteristics of solid lipid nanoparticles (SLNs). Biomaterials 24:1781-1785

Hu FQ, Jiang SP et al (2006) Preparation and characteristics of monostearin nanostructured lipid carriers. Int J Pharm 314 (1):83-89

Jemal A, Murray T et al (2005) Cancer statistics. Cancer J Clin 55:10-30

Li F, Weng Y et al (2010) The efficacy and safety of bufadienolidesloaded nanostructured lipid carriers. Int J Pharm 393(1-2):203-211

Liu D, Liu Z et al (2011) Nanostructured lipid carriers as novel carrier for parenteral delivery of docetaxel. Colloids and Surfaces B: Biointerfaces 85:262-269

Liu Y, Lu WL et al (2007) Controlled delivery of recombinant hirudin based on thermo-sensitive Pluronic F127 hydrogel for subcutaneous administration: in vitro and in vivo characterization. J Control Release 117(3):387-395

Luciano O, De Rosa CM, Kari C, Hanna-Leena K, Habif DV Sr (1995) Regression of human breast cancer xenografts in response to intralesional treatment with interferons $\alpha$ and $\gamma$ potentiated by tumor necrosis factor. J Interferon Cytokine Res 15(10):839-848

Matsumura Y, Maeda H (1986) A new concept for macromolecular therapeutics in cancer chemotherapy: mechanism of tumoritropic accumulation of proteins and the antitumor agent SMANCS. Cancer Res 46(12 Pt 1):6387-6392

Mendes S, Graziani SR, Vitório TS, Padoveze AF, Hegg R, Bydlowski SP, Maranhão RC (2009) Uptake by breast carcinoma of a lipidic nanoemulsion after intralesional injection into the patients: a new strategy for neoadjuvant chemotherapy. Gynecol Oncol 112 (2):400-404

Muller RH, Petersen RD et al (2007) Nanostructured lipid carriers (NLC) in cosmetic dermal products. Adv Drug Deliv Rev 59 (6): $522-530$

Paola SM, Houria B, Juan LO, Jose MP, Antonia A (2012) Novel drug delivery system based on docetaxel loaded nanocapsules as a therapeutic strategy against breast cancer cells. Int J Mol Sci 13 (4):4906-4919

Pople PV, Singh KK (2011) Development and evaluation of colloidal modified nanolipid carrier: application to topical delivery of tacrolimus. Eur J Pharm Biopharm 79(1):82-94

Rob C, Loanna P et al (2006) Overcoming physiologic barriers to cancer treatment by molecularly targeting the tumor microenvironment. Mol Cancer Res 4(2):61-70

Rouzier R, Extra J-M et al (2001) Primary chemotherapy for operable breast cancer: incidence and prognostic significance of ipsilateral breast tumor recurrence after breast conserving surgery. J Clin Oncol 19:3828-3835

Souza LG, Silva EJ et al (2011) Development of topotecan loaded lipid nanoparticles for chemical stabilization and prolonged release. Eur J Pharm Biopharm 79(1):189-196

Wang PL, Johnston TP (1995) Sustained-release interleukin-2 following intramuscular injection in rats. Int J Pharm 113(1):73-81

Westesen K, Bunjes H et al (1997) Physicochemical characterization of lipid nanoparticles and evaluation of their drug loading capacity and sustained release potential. J Controlled Release 48:223-236

Xu Z, Chen L, Gu W, Gao Y, Lin L, Zhang Z, Xi Y, Li Y (2009) The performance of docetaxel-loaded solid lipid nanoparticles targeted to hepatocellular carcinoma. Biomaterials 30(2):226-232

Yang Y, Wang J et al (2009) A novel mixed micelle gel with thermosensitive property for the local delivery of docetaxel. J Control Release 135(2):175-182 